\title{
Comme la centralité, la ruralité a un coût
}

\author{
Jacques D esbiens \\ Université du Q uébec à Chicoutimi
}

\section{Introduction}

On fait généralement l'hypothèse que la ruralité coûte cher sous l'angle des services municipaux offerts. En effet, comme nous le verrons un peu plus loin dans l'analyse de nos données, plus une municipalité a un caractère rural, plus sa taille est petite. Or, comme nous le signale Dugas ${ }^{1}$, «plus la taille démographique d'une localité est petite, plus sa base de services est ténue. Ce constat sommaire conduit de nombreux observateurs et analystes à suggérer des regroupements administratifs de population afin d'élargir, de diversifier et d'améliorer l'infrastructure des services et de diminuer son coût d'utilisation per capita. Une telle proposition, qui s'inspire du principe des économies d'échelle, alimente depuis au moins le début des années 1960 un courant d'idées favorable aux fusions ou aux regroupements des petites municipalités du Québec.» Nous avons déjà nousmêmes ${ }^{2}$ constaté que «les fusions et les regroupements de municipalités sont susceptibles de réduire les coûts des municipalités dont la taille est inférieure à 2000 habitants » au Québec.

Cette relation avec la petite taille sert souvent de base à la définition de la ruralité. Ainsi, Dugas ${ }^{3}$ précise que «Statistique Canada définit comme rural tout ce qui n'est pas urbain. Est considéré comme urbain tout groupement de 1000 habitants et plus qui a une densité d'au moins 400 habitants au kilomètre carré. Pour mieux circonscrire les territoires à dominance rurale, il est d'usage d'utiliser des seuils démographiques en plus de ces critères de Statistique Canada. Les chiffres de 2500 ou 3000 habitants sont généralement retenus.»
En ce qui nous concerne, comme nous le verrons dans la partie méthodologique qui suit, nous avons plutôt opté pour l'approche continuum urbain - rural que propose entre autres Bruneau ${ }^{4}$. Pour lui, «À l'opposition des termes ville - campagne succède le continuum urbain - rural, dont le point d'origine est le pôle urbain et qui structure quatre types d'espace : 1) la zone périurbaine; 2) la frange urbaine; 3) la zone périphérique; 4) la zone rurale traditionnelle. La première couronne urbaine (la zone périurbaine) est contiguë au pole central. C'est au-delà de la première couronne dite «périurbaine» que se développe la frange urbaine. Au-delà de cette deuxième couronne urbaine, il y a apparition de deux autres types d'espace : celui où l'exode rural est encore actif et, donc, toujours sous influence urbaine, et un autre, plus lointain, où le rural, après avoir cédé à la ville l'essentiel de ses forces vives, fait figure de survivance.»

Statistique Canada définit comme rural tout ce qui n'est pas urbain. Est considéré comme urbain tout groupement de 1000 habitants et plus qui a une densité d'au moins 400 habitants au kilomètre carré. Pour mieux circonscrire les territoires à dominance rurale, il est d'usage d'utiliser des seuils démographiques en plus de ces critères de Statistique Canada. Les chiffres de 2500 ou 3000 habitants sont généralement retenus. 


\section{Méthodologie}

De façon à vérifier l'hypothèse que la ruralité coûte cher, nous avons examiné l'impact du degré de ruralité sur certains indicateurs de coût des services municipaux. À partir des travaux sur le continuum urbain - rural, nous avons classé les municipalités locales selon quatre degrés de ruralité ${ }^{5}$ :

1) Degré 0 : l'agglomération urbaine On y trouve les municipalités locales faisant partie d'une région métropolitaine ou d'une agglomération de recensement;

2) Degré 1 : la zone périurbaine Elle comprend les municipalités locales adjacentes à l'agglomération urbaine;

3) Degré 2 : la frange urbaine Elle est constituée des municipalités locales non adjacentes à l'agglomération urbaine, faisant partie de la première couronne après les municipalités adjacentes;

4) Degré 3 : la zone périphérique

Elle comprend les municipalités locales non adjacentes à l'agglomération urbaine, faisant partie de la seconde couronne après les municipalités adjacentes.

Nous avons examiné les relations entre le degré de ruralité ainsi mesuré et certains indicateurs de coût des services municipaux, mesurant les trois aspects suivants de l'offre de service :

\section{1) Les dépenses d'administration générale}

- les dépenses nettes ${ }^{6}$ d'administration générale par habitant en 1998;

- les dépenses nettes d'administration générale par $1000 \$$ de richesse foncière uniformisée en 1998;

- les dépenses nettes d'administration générale en pourcentage des dépenses totales nettes ${ }^{7}$ de la municipalité en 1998.

2) Les dépenses totales et la facture totale

- les dépenses totales nettes de la municipalité par habitant en 1998;

- les dépenses totales nettes de la municipalité par $1000 \$$ de richesse foncière uniformisée en 1998;
- le taux global de taxation uniformisé (TGTU) en 1998.

3) Le fardeau de la dette

- les dépenses nettes ${ }^{8}$ de frais de financement du service de la dette à la charge de la municipalité en pourcentage des dépenses totales nettes de la municipalité en 1998;

- l'endettement total net à long terme en pourcentage de la richesse foncière uniformisée en 1998.

De façon à percevoir la présence d'économies d'échelle potentielles dans les municipalités de notre échantillon, nous avons également examiné les liens entre ces indicateurs de coût et les variables suivantes :

1) la population de la municipalité locale en 1998;

2) la richesse foncière uniformisée sur le territoire de la municipalité en 1998;

3) la superficie de la municipalité en 1998;

4) la densité de la population au kilomètre carré dans la municipalité en 1998.

Nous avons choisi un échantillon de convenance de 142 municipalités provenant des $9 \mathrm{MRC}$ suivantes où l'on trouve une agglomération urbaine de plus de 10000 habitants.

\section{Thinen 1. Ia MrC taridited Ine notre fuimtilln}

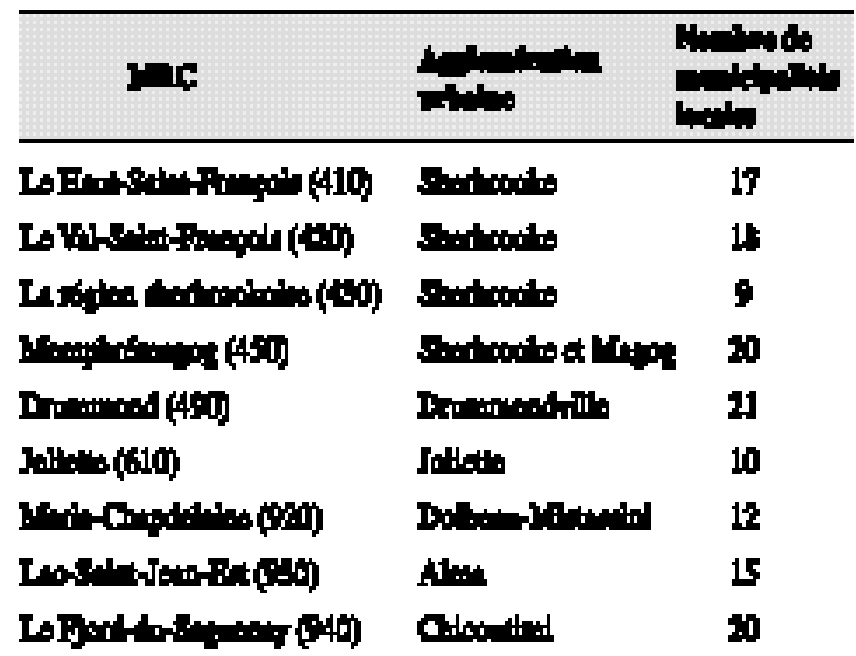


Trente-neuf de ces municipalités locales font partie d'une agglomération urbaine, quarante-cinq se situent en zone périurbaine, trente-huit sont dans la frange urbaine, et vingt se trouvent dans la zone périphérique.

\section{Analyse des résultats ${ }^{9}$}

\section{L'impact du degré de ruralité}

\section{Les dépenses d'administration générale} selon le degré de ruralité

Nous avons constaté une différence de moyenne statistiquement significative ${ }^{10}$ entre les dépenses d'administration générale par habitant des municipalités locales considérées, selon leur degré de ruralité. En fait, plus le degré de ruralité est élevé, plus grandes tendent à être les dépenses d'administration générale par habitant, en moyenne. Elles passent de 122,14 \$ en moyenne dans les 39 municipalités faisant partie d'une agglomération urbaine, à 143,50 \$ dans les 45 zones périurbaines, puis à 159,34 \$ dans les 37 municipalités de la frange urbaine, avant de culminer à 174,88 \$ en moyenne dans les 19 zones périphériques.

Nous avons constaté une différence de moyenne statistiquement significative entre les dépenses d'administration générale par habitant des municipalités locales considérées, selon leur degré de ruralité. En fait, plus le degré de ruralité est élevé, plus grandes tendent à être les dépenses d'administration générale par habitant, en moyenne.

Il y a également une différence de moyenne statistiquement significative ${ }^{11}$ entre les dépenses d'administration générale par $1000 \$$ de richesse foncière des municipalités locales considérées, selon leur degré de ruralité. En fait, là aussi, plus le degré de ruralité est élevé, plus grandes tendent à être les dépenses d'administration générale en fonction de la richesse foncière, en moyenne. Les 19 zones périphériques ont, en moyenne, comme dans le cas précédent, des dépenses supérieures $(5,16 \$)$ à celles des 37 municipalités de la frange urbaine $(5,02 \$)$. Les 45 zones périurbaines ne dépensent que 3,98 \$ en moyenne par
$1000 \$$ de richesse foncière pour l'administration générale, tandis que ce montant ne s'élève qu'à 3,28 \$ dans les 39 municipalités situées dans des agglomérations urbaines de notre échantillon.

Notre troisième indicateur de mesure des dépenses d'administration générale varie lui aussi en fonction du degré de ruralité. Ainsi, nous remarquons, encore une fois, une différence de moyenne statistiquement significative $^{12}$ entre les pourcentages des dépenses totales consacrées à l'administration générale des municipalités locales considérées, selon leur degré de ruralité. Plus le degré de ruralité est élevé, plus ce pourcentage tend à être élevé, en moyenne. Il n'est que de $18,31 \%$ en moyenne dans nos 39 municipalités faisant partie d'une agglomération urbaine, mais s'élève déjà à $28,11 \%$ dans les 45 zones périurbaines. Les municipalités de la zone périphérique consacrent cependant, quant à elles, en moyenne, un pourcentage de leurs dépenses totales à l'administration générale légèrement inférieur $(30,62 \%)$ à celui des municipalités de la frange urbaine $(32,19 \%)$.

\section{Les dépenses totales et la facture totale de la municipalité selon le degré de ruralité}

On observe encore une fois une différence de moyenne statistiquement significative ${ }^{13}$ entre les dépenses totales de la municipalité par habitant dans nos municipalités, selon leur degré de ruralité. Cette fois cependant, les résultats sont un peu différents. Ainsi, les dépenses totales par habitant sont d'abord particulièrement élevées en moyenne dans les 39 municipalités faisant partie d'une agglomération urbaine $(714,60 \$)$. Elles chutent par la suite à 545,06 \$ en moyenne dans les 45 zones périurbaines, mais s'accroissent systématiquement par la suite avec le caractère rural. Ainsi, elles augmentent en moyenne à 550,62 \$ dans les 37 franges urbaines puis s'élèvent à 593,73 \$ dans les 19 zones périphériques.

On observe une différence de moyenne statistiquement significative entre les dépenses totales de la municipalité par habitant dans nos municipalités, selon leur degré de ruralité. 
On constate aussi une différence de moyenne statistiquement significative ${ }^{14}$ entre les dépenses totales de la municipalité par $1000 \$$ de richesse foncière uniformisée dans les municipalités que nous examinons, selon leur degré de ruralité. On observe, dans ce cas, le même type de relation que celle que nous venons de décrire concernant les dépenses totales par habitant. Ici encore, les dépenses totales de la municipalité par $1000 \$$ de richesse foncière uniformisée sont particulièrement élevées en moyenne dans les 39 municipalités situées dans les agglomérations urbaines (19,24 \$). Par la suite, elles diminuent dans les 45 zones périurbaines (14,51 \$) avant d'augmenter régulièrement dans les 37 franges urbaines $(15,98 \$)$ puis dans les 19 zones périphériques $(18,85 \$)$.

Enfin, on observe une fois de plus une différence de moyenne statistiquement significative ${ }^{15}$ entre le taux global de taxation uniformisé de nos municipalités, selon leur degré de ruralité. Ce taux est, encore une fois, particulièrement élevé d'abord en moyenne $(1,60)$ dans les $38^{16}$ municipalités faisant partie d'une agglomération urbaine. Puis il diminue fortement en moyenne $(1,25)$ dans les 45 municipalités faisant partie de la zone périurbaine. Il augmente ensuite régulièrement en moyenne dans les $36^{17}$ municipalités $(1,36)$ faisant partie de la frange urbaine de même que dans les $18^{18}$ municipalités $(1,53)$ de la zone périphérique.

\section{Le fardeau de la dette selon le degré de ruralité}

Il y a encore une fois une différence de moyenne statistiquement significative ${ }^{19}$ entre le pourcentage que représentent les frais de financement de la dette sur les dépenses totales des 140 municipalités locales considérées, selon leur degré de ruralité. Ce pourcentage est très élevé d'abord en moyenne $(15,14 \%)$ dans les 39 municipalités situées dans les agglomérations urbaines. Puis il diminue beaucoup en moyenne $(6,19 \%)$ dans les 45 zones périurbaines et dans les 37 municipalités $(5,98 \%)$ faisant partie de la frange urbaine. Il remonte ensuite légèrement dans les 19 zones périphériques $(7,65 \%)$.

Une fois de plus, on constate une différence de moyenne statistiquement significative ${ }^{20}$ entre l'endettement total net à long terme en pourcentage de la richesse foncière uniformisée dans les municipalités que nous examinons, selon leur degré de ruralité. On observe, dans ce cas, le même type de relation que celle que nous avons décrite dans la section sur les dépenses totales et la facture totale, c'est-à-dire que le pourcentage d'endettement en fonction de la richesse foncière est particulièrement élevé en moyenne dans les 39 municipalités membres d'une agglomération urbaine $(2,35 \%)$, avant de diminuer fortement dans les 45 zones périurbaines $(1,03 \%)$ et d'augmenter graduellement par la suite dans les 37 franges urbaines $(1,21 \%)$, puis dans les 19 zones périphériques $(1,48 \%)$.

Il y a une différence de moyenne statistiquement significative entre le pourcentage que représentent les frais de financement de la dette sur les dépenses totales des 140 municipalités locales considérées, selon leur degré de ruralité.

\section{L'influence des autres variables sur les indicateurs de coût}

Si le degré de ruralité affecte nos indicateurs de coût des services municipaux, ce n'est cependant pas la seule, ni nécessairement la plus importante variable qui a un impact sur eux. En effet, l'examen de nos données nous a également révélé d'autres liens intéressants.

\section{Les autres variables affectant les dépenses d'administration générale}

Nous avons observé une relation statistiquement significative $^{21}$ entre la population et les dépenses d'administration générale par habitant. Ces dépenses ont tendance à diminuer de manière curviligne lorsque la population est plus élevée dans les 124 municipalités de notre échantillon ayant moins de 6000 habitants. On est donc en présence d'un phénomène d'économies d'échelle ici. On constate aussi une relation statistiquement significative ${ }^{22}$ entre la densité de la population et les dépenses d'administration générale par habitant. Ces dépenses ont également tendance à diminuer de manière curviligne lorsque la densité augmente dans les 115 municipalités de notre échantillon ayant moins de 100habitants par kilomètre carré. Par ailleurs, la superficie n'est pas apparue reliée de manière statistiquement significative aux 
dépenses d'administration générale par habitant dans les municipalités de notre échantillon.

Nous avons observé une relation statistiquement significative entre la population et les dépenses d'administration générale par habitant. On constate aussi une relation statistiquement significative entre la densité de la population et les dépenses d'administration générale par habitant.

Il y a également une relation statistiquement significative $^{23}$ entre la richesse foncière uniformisée et les dépenses d'administration générale par $1000 \$$ de richesse foncière uniformisée. Ces dépenses ont tendance à diminuer de manière curviligne lorsque la richesse foncière est plus élevée dans les 122 municipalités de notre échantillon ayant moins de 200 millions de dollars de richesse foncière uniformisée. Là aussi, on peut donc parler d'économies d'échelle. On observe encore une relation statistiquement significative ${ }^{24}$ entre la densité de la population et les dépenses d'administration générale par $1000 \$$ de richesse foncière uniformisée. Ces dépenses ont également tendance à diminuer de manière curviligne lorsque la densité de la population est plus élevée dans les 107 municipalités de notre échantillon ayant moins de 60 habitants par kilomètre carré.

Ici non plus, nous n'avons cependant pas observé de relation statistiquement significative entre la superficie et les dépenses d'administration générale par 1000 \$ de richesse foncière dans les municipalités de notre échantillon.

La part que représentent les dépenses d'administration générale sur les dépenses totales des municipalités de notre échantillon varie de manière statistiquement significative $^{25}$ avec la taille des municipalités mesurée par la population. Ce pourcentage a tendance à diminuer de manière curviligne lorsque la population est plus élevée dans les 128 municipalités de notre échantillon ayant moins de 10000 habitants. On observe le même type de relation statistiquement significative ${ }^{26}$ entre le pourcentage que représentent les dépenses d'administration générale sur les dépenses totales des municipalités de notre échantillon et la taille des municipalités mesurée par la richesse foncière uniformisée. Là aussi, ce pourcentage a tendance à diminuer de manière curviligne lorsque la richesse foncière augmente dans les 122 municipalités de notre échantillon ayant moins de 200 millions de dollars de richesse foncière. La densité de la population est aussi reliée de manière statistiquement significative ${ }^{27}$ au pourcentage que représentent les dépenses d'administration générale sur les dépenses totales des municipalités de notre échantillon. Une fois de plus, ce pourcentage a tendance à diminuer de manière curviligne lorsque la densité augmente dans les 115 municipalités de notre échantillon ayant moins de 100 habitants au kilomètre carré.

\section{Les autres variables affectant les dépenses totales et la facture totale des municipalités}

À part le degré de ruralité, aucune autre des variables que nous avons considérées, soit la population, la richesse foncière, la superficie et la densité, n'est apparue reliée aux dépenses totales par habitant dans les municipalités de notre échantillon.

Comme pour les dépenses totales par habitant, aucune autre des variables que nous avons considérées en plus du degré de ruralité, soit la population, la richesse foncière, la superficie et la densité n'est apparue reliée aux dépenses totales par $1000 \$$ de richesse foncière dans les municipalités de notre échantillon.

À part le degré de ruralité, aucune autre des variables que nous avons considérées, soit la population, la richesse foncière, la superficie et la densité, n'est apparue reliée aux dépenses totales par habitant dans les municipalités de notre échantillon.

Enfin, en ce qui concerne le taux global de taxation uniformisé (TGTU), là non plus, à part le degré de ruralité, nous n'avons pas observé de relation digne de confiance avec aucune autre des variables que nous avons considérées, soit la population, la richesse foncière, la superficie et la densité. 


\section{Les autres variables affectant le fardeau de la dette}

En ce qui concerne le pourcentage que représentent les frais de financement sur les dépenses totales de la municipalité, trois variables y sont apparues reliées de manière statistiquement significative, outre le degré de ruralité, soit la population ${ }^{28}$, la richesse foncière uniformisée $^{29}$ et la densité de la population ${ }^{30}$. Dans les trois cas, la relation est la même : elle est linéaire et positive. En somme, les frais de la dette en pourcentage des dépenses totales sont plus élevés dans nos municipalités locales lorsque la population est plus grande, la richesse foncière plus considérable et la densité de la population plus forte.

En ce qui concerne le pourcentage que représentent les frais de financement sur les dépenses totales de la municipalité, trois variables y sont apparues reliées de manière statistiquement significative, outre le degré de ruralité, soit la population, la richesse foncière uniformisée et la densité de la population. En somme, les frais de la dette en pourcentage des dépenses totales sont plus élevés dans nos municipalités locales lorsque la population est plus grande, la richesse foncière plus considérable et la densité de la population plus forte.

En ce qui concerne l'endettement total net à long terme en pourcentage de la richesse foncière uniformisée, à part le degré de ruralité, aucune autre des variables que nous avons considérées, soit la population, la richesse foncière, la superficie et la densité, n'y est vraiment reliée de façon claire et nette.

\section{Conclusion}

Nous venons de le voir, la ruralité a un coût en matière de services municipaux offerts. Entre autres choses, le fait d'avoir un caractère plus rural augmente la facture et le fardeau de l'administration générale. En effet, les dépenses d'administration générale par habitant ou par $1000 \$$ de richesse foncière uniformisée tendent systématiquement à être plus élevées lorsque le degré de ruralité d'une municipalité locale s'accroît. Il en va de même avec le pourcentage que représentent les dépenses d'administration générale sur les dépenses totales de la municipalité.

Par ailleurs, en ce qui concerne les dépenses totales, la facture totale et l'endettement, nos conclusions sont à deux volets. D'abord, le fait de faire partie d'une agglomération urbaine occasionne des dépenses particulièrement élevées et entraîne un lourd fardeau d'endettement. Cependant, ces dépenses et le poids de la dette chutent de manière radicale lorsqu' on est en zone périurbaine, avant de remonter par la suite avec le degré de ruralité.

En effet, à l'exception des municipalités membres d'une agglomération urbaine où ces dépenses sont particulièrement élevées, les dépenses totales, qu'on les mesure par habitant ou en fonction de la richesse foncière, s'élèvent avec la ruralité d'une municipalité.

Le fardeau fiscal global varie de la même manière. C'est ce que nous avons constaté avec le TGTU. Effectivement, il est d'abord particulièrement élevé dans les municipalités locales membres d'une agglomération urbaine. Quant aux autres municipalités locales de notre échantillon, nous avons bien vu qu'il augmente systématiquement avec le degré de ruralité.

Nous avons observé la même situation avec l'endettement. En effet, encore une fois en excluant les municipalités locales membres d'une agglomération urbaine où le fardeau de la dette est particulièrement lourd, la charge financière de la dette augmente, plus une municipalité est rurale. Nous avons constaté ce phénomène en partie avec le pourcentage des frais de financement sur les dépenses totales, mais particulièrement avec la dette en pourcentage de la richesse foncière uniformisée.

En ce qui concerne la population et la richesse foncière, ces deux variables font baisser les coûts unitaires des services municipaux, comme nous l'avons vu, puisque nous observons des phénomènes d'économies d'échelle dans nos municipalités locales de moins de 6000 habitants ou de moins de 200 millions de dollars de richesse foncière uniformisée. Or, la population et la richesse foncière sont fortement reliées en sens inverse avec le degré de ruralité. En effet, la population et la richesse foncière uniformisée 
tendent à diminuer de manière systématique avec le degré de ruralité. Une autre indication que la ruralité coûte généralement plus cher dans les municipalités locales au Québec.

\section{Notes et références}

1 DUGAS, Clermont (1994). «Le regroupement des services en milieu rural», Relations, $\mathrm{n}^{\mathrm{O}} 605$, novembre, p. 279.

2 DESBIENS, Jacques (1999). Fusions municipales et économies d'échelle: Mythes et réalités, Chicoutimi, Le groupe Jacques Desbiens, p. 90.

3 DUGAS, Clermont (1994). Op. cit., note 1, p. 280.

4 BRUNEAU, Pierre (2000). Le Québec en changement, SainteFoy, PUQ, p. 38-42.

5 Aucune des municipalités de notre échantillon ne se situant dans cette zone, nous n'avons pas considéré le type rural profond.

6 Nettes des autres recettes de source locale pour services rendus aux organismes municipaux (administration générale).

7 Les dépenses totales nettes sont obtenues en soustrayant des dépenses totales de la municipalité les éléments suivants : 1) les dépenses nettes d'électricité; 2) le total des autres recettes de sources locales pour services rendus aux organismes municipaux; 3) le total des autres recettes de sources locales pour autres services rendus, déduction faite du total des recettes des autres services rendus pour l'électricité; 4) le total des recettes de transferts à l'exclusion des subventions du gouvernement du Québec pour compensation de plafonnement et des subventions du gouvernement du Québec pour compensation des droits sur les divertissements.

8 Nettes des autres recettes de source locale pour services rendus aux organismes municipaux (frais de financement).

9 Les données que nous avons analysées dans ce travail proviennent des fichiers informatisés intitulés Finances des organismes municipaux pour l'exercice financier 1998, édition 2000, des Publications du Québec, ainsi que des fichiers informatisés intitulés Répertoire des municipalités $d u$ Québec 1998, également des Publications du Québec.

10 Analyse de variance à un facteur : $\mathrm{n}=140, \mathrm{~F}=4,73$ et $\mathrm{p}=0,0036$

11 Analyse de variance à un facteur : $\mathrm{n}=140, \mathrm{~F}=3,91$ et $\mathrm{p}=0,0103$

12 Analyse de variance à un facteur : $\mathrm{n}=140, \mathrm{~F}=11,87$ et $\mathrm{p}=0,0001$
13 Analyse de variance à un facteur : $\mathrm{n}=140, \mathrm{~F}=3,84$ et $\mathrm{p}=0,0111$

14 Analyse de variance à un facteur : $\mathrm{n}=140, \mathrm{~F}=3,04$ et $\mathrm{p}=0,0314$

15 Analyse de variance à un facteur : $\mathrm{n}=140, \mathrm{~F}=3,61$ et $\mathrm{p}=0,0151$

16 Bromptonville ayant fait l'objet d'une fusion, nous ne disposions pas de son TGTU pour 1998.

17 Métabetchouan - Lac-à-la-Croix ayant fait l'objet d'une fusion, nous ne disposions pas de son TGTU pour 1998.

18 Richmond ayant fait l'objet d'une fusion, nous ne disposions pas de son TGTU pour 1998.

19 Analyse de variance à un facteur : $\mathrm{n}=140, \mathrm{~F}=14,6$ et $\mathrm{p}=0,0001$

20 Analyse de variance à un facteur : $\mathrm{n}=140, \mathrm{~F}=7,3$ et $\mathrm{p}=0,0001$

21 Régression polynomiale du second degré $: \mathrm{n}=124, \mathrm{~F}=16,62$, $\mathrm{p}=0.0001, \mathrm{r}^{2}=0,22$

22 Régression polynomiale du second de gré : $\mathrm{n}=115, \mathrm{~F}=14,6$, $\mathrm{p}=0.0001, \mathrm{r}^{2}=0,21$

23 Régression polynomiale du second degré $: \mathrm{n}=122, \mathrm{~F}=24,62$, $\mathrm{p}=0.0001, \mathrm{r}^{2}=0,29$

24 Régression polynomiale du second degré : $\mathrm{n}=107, \mathrm{~F}=7,66$, $\mathrm{p}=0.0008, \mathrm{r}^{2}=0,13$

25 Régression polynomiale du second degré $: \mathrm{n}=128, \mathrm{~F}=29,11$, $\mathrm{p}=0.0001, \mathrm{r}^{2}=0,32$

26 Régression polynomiale du second degré $: \mathrm{n}=122, \mathrm{~F}=26,57$, $\mathrm{p}=0.0001, \mathrm{r}^{2}=0,31$

27 Régression polynomiale du second degré $: \mathrm{n}=115, \mathrm{~F}=22,68$, $\mathrm{p}=0.0001, \mathrm{r}^{2}=0,29$

28 Régression linéaire simple $: \mathrm{n}=124, \mathrm{~F}=53,74, \mathrm{p}=0,0001$ et $r^{2}=0,31$

29 Régression linéaire simple $: \mathrm{n}=127, \mathrm{~F}=33,79, \mathrm{p}=0,0001$ et $r^{2}=0,21$

30 Régression linéaire simple $: \mathrm{n}=137, \mathrm{~F}=67,27, \mathrm{p}=0,0001$ et $r^{2}=0,33$ 